\title{
Collimated blue and infrared beams generated by two-photon excitation in $\mathbf{R b}$ vapor
}

\author{
J. F. Sell, ${ }^{1, *}$ M. A. Gearba, ${ }^{1,2}$ B. D. DePaola, ${ }^{3}$ and R. J. Knize ${ }^{1}$ \\ ${ }^{1}$ Laser and Optics Research Center, Department of Physics, U.S. Air Force Academy, Colorado 80840, USA \\ ${ }^{2}$ Department of Physics and Astronomy, University of Southern Mississippi, Hattiesburg, Mississippi 39406, USA \\ ${ }^{3}$ Department of Physics, Kansas State University, Manhattan, Kansas 66506, USA \\ ${ }^{*}$ Corresponding author: jerry.sell.1@gmail.com
}

Received November 12, 2013; accepted November 26, 2013;

posted December 13, 2013 (Doc. ID 201185); published January 23, 2014

Utilizing two-photon excitation in hot Rb vapor we demonstrate the generation of collimated optical fields at 420 and $1324 \mathrm{~nm}$. Input laser beams at 780 and $776 \mathrm{~nm}$ enter a heated Rb vapor cell collinear and circularly polarized, driving $\mathrm{Rb}$ atoms to the $5 D_{5 / 2}$ state. Under phase-matching conditions coherence among the $5 S_{1 / 2} \rightarrow 5 P_{3 / 2} \rightarrow 5 D_{5 / 2} \rightarrow$ $6 P_{3 / 2}$ transitions produces a blue $(420 \mathrm{~nm})$ beam by four-wave mixing. We also observe a forward and backward propagating IR (1324 nm) beam, due to cascading decays through the $6 S_{1 / 2} \rightarrow 5 P_{1 / 2}$ states. Power saturation of the generated beams is investigated by scaling the input powers to greater than $200 \mathrm{~mW}$, resulting in a coherent blue beam of $9.1 \mathrm{~mW}$ power, almost an order of magnitude larger than previously achieved. We measure the dependences of both beams in relation to the Rb density, the frequency detuning between $\mathrm{Rb}$ ground-state hyperfine levels, and the input laser intensities. (c) 2014 Optical Society of America

OCIS codes: (190.4223) Nonlinear wave mixing; (190.7220) Upconversion.

http://dx.doi.org/10.1364/OL.39.000528

A wide range of phenomena can be created by exploiting nonlinear optical processes in a dense atomic vapor. Large enhancements of these processes are possible through the generation of quantum coherences among atomic states and include effects, such as electromagnetically induced transparency, fast and slow light propagation, four-wave mixing (FWM), and lasing without inversion. FWM in particular has been shown to produce both efficient frequency upconversion [1-3] and downconversion [4] using low-power continuous-wave (cw) lasers. The newly created optical fields are narrowband tunable coherent light sources [5], with wavelengths from the IR to approaching the UV depending upon the atomic states involved.

Frequency upconversion by FWM has most often been studied in Rb vapor, first demonstrated using low-power cw lasers by Zibrov et al. in 2002 [6] where $15 \mu \mathrm{W}$ of coherent radiation at $420 \mathrm{~nm}$ was achieved. The method relies upon input lasers at 780 and $776 \mathrm{~nm}$ [see Fig. 1(a)] driving $\mathrm{Rb}$ atoms from the $5 S_{1 / 2}$ ground state to the $\overline{5 D_{5 / 2}}$ state by two-photon excitation, with the $5 P_{3 / 2}$ level as an intermediate state. A third optical field between the $5 D_{5 / 2} \rightarrow 6 P_{3 / 2}$ levels at $5.23 \mu \mathrm{m}$ is initiated through spontaneous emission. Strong atomic coherences are thus formed in a diamond-type energy level structure, creating coherent blue light at $420 \mathrm{~nm}\left(6 P_{3 / 2} \rightarrow 5 S_{1 / 2}\right)$ by FWM. Recent experiments achieved first $40 \mu \mathrm{W}$ of $420 \mathrm{~nm}$ light through the additional coupling of both $5 S_{1 / 2}$ hyperfine ground-state levels [1] , and subsequently $1.1 \mathrm{~mW}$ by further optimization of input laser polarizations and frequencies [3]. The generated blue beam exhibits a high degree of spatial coherence [2], with a spectral linewidth typically limited by the linewidths of the applied laser fields [7]. The absolute frequency of the blue light has been found to be centered on the $6 P_{3 / 2} \rightarrow 5 S_{1 / 2}$ transition, with tunability of $\geq 100 \mathrm{MHz}$ possible by adjustment of the input laser frequencies [5]. Incorporating an additional laser at $795 \mathrm{~nm}$ has been shown to both enhance and suppress the FWM process through control of optical pumping [8]. A single-frequency laser at $778 \mathrm{~nm}$ has also been shown to produce blue $(420 \mathrm{~nm})$ beams, using both cw [9] and pulsed excitation [10]. Utilizing the corresponding two-step excitation scheme in Cs, $4 \mu \mathrm{W}$ of coherent blue light was generated at $455 \mathrm{~nm}$ [11], illustrating the application of this method to less ideal cases as only $0.4 \%$ of Cs atoms cascade from $6 D_{5 / 2} \rightarrow 7 P_{3 / 2}$ compared to $35 \%$ of $\mathrm{Rb} 5 D_{5 / 2}$ atoms, which decay through the $6 P_{3 / 2}$ state [12].

In this Letter, we analyze the collimated beams at 420 and $1324 \mathrm{~nm}$ that are produced during two-photon excitation to the $\mathrm{Rb} 5 D_{5 / 2}$ state. Substantially greater blue beams than previously observed are achieved by scaling the input beams to much higher intensities while adjusting their frequency detunings along with the $\mathrm{Rb}$ density in order to obtain optimal phase matching and input beam absorption for blue beam generation and transmission. The coherent and collimated blue light can be used as a basis for applications, such as sensitive atom
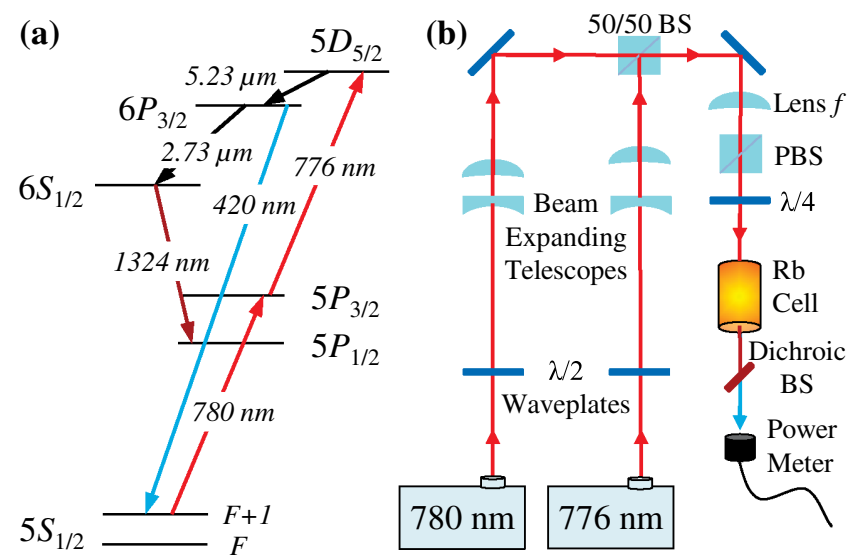

Fig. 1. (a) Relevant Rb energy levels. Excitation lasers (cw) are present at 780 and $776 \mathrm{~nm}$ while collimated output beams are observed at 420 and $1324 \mathrm{~nm}$. (b) Schematic of the experimental setup. 
detection [13], quantum information processing [14], and underwater communication [10]. The generated $1324 \mathrm{~nm}$ beam has seldomly been studied; however, we find it can be significant reaching levels of $2.5 \mathrm{~mW}$ or more. The forward and backward propagating $1324 \mathrm{~nm}$ beam can occur independently or simultaneously with the generated blue beam, which can help to characterize the coherence and population dynamics occurring during the FWM process.

The experimental setup is illustrated schematically in Fig. 1(b). A master oscillator and tapered amplifier laser system drives the $D_{2}$ transition in Rb at $780 \mathrm{~nm}$, while a titanium:sapphire ring laser drives the $5 P_{3 / 2} \rightarrow 5 D_{5 / 2}$ transition at $776 \mathrm{~nm}$. The two laser beams are combined in a 50/50 nonpolarizing beam splitter and pass through a $\lambda / 4$ wave plate in order to achieve copropagating and circularly polarized input optical fields, whose relevance in coherent blue light generation has been identified in previous experiments [1, $\underline{3}]$. An $f=250 \mathrm{~mm}$ lens is used to focus the laser beams to a waist radius of $\simeq 100 \mu \mathrm{m}$ in the vapor cell, resulting in intensities of up to $10^{3} \mathrm{~W} / \mathrm{cm}^{2}$ (350 $\mathrm{mW}$ power) in each laser beam. This choice was made to allow a depth of focus approximately the length of the vapor cell $(5 \mathrm{~cm})$. The $\mathrm{Rb}$ vapor cell contains a natural abundance of $\mathrm{Rb}$ and is operated from $105^{\circ} \mathrm{C}$ to $135^{\circ} \mathrm{C}\left(8 \times 10^{12}-5 \times 10^{13} \mathrm{~cm}^{-3}\right)$. Beam expanding telescopes are also incorporated into each laser beam path to optimally overlap the beams in the $\mathrm{Rb}$ vapor cell. We measure the power of the generated beams after they pass through a dichroic beam splitter which reflects $\geq 99.8 \%$ of the light from 776 to $780 \mathrm{~nm}$, while transmitting $\geq 75 \%$ at 420 and $1324 \mathrm{~nm}$. Possible beam emissions at 2.7 and $5.2 \mu \mathrm{m}$ are not observed as they do not transmit through the windows of the vapor cell.

Figure 2 illustrates the coherent $420 \mathrm{~nm}$ power generated as a function of the $780 \mathrm{~nm}$ laser detuning from the ${ }^{85} \mathrm{Rb} 5 S_{1 / 2}(F=3) \rightarrow 5 P_{3 / 2}\left(F^{\prime}\right)$ absorption resonance. A Burleigh WA-1500 wavemeter (resolution of $0.001 \mathrm{~cm}^{-1} \simeq 30 \mathrm{MHz}$ ) is used to record the $780 \mathrm{~nm}$ laser frequency, which is calibrated by scanning across $\mathrm{Rb}$ absorption features in a room-temperature vapor cell

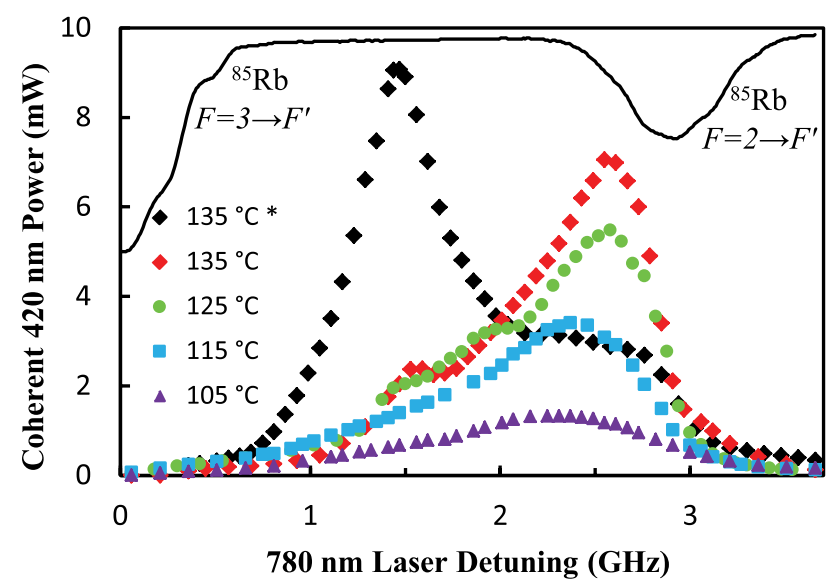

Fig. 2. Generated blue beam power as a function of the $780 \mathrm{~nm}$ laser frequency, referenced to ${ }^{85} \mathrm{Rb}$ ground hyperfine state absorption peaks (shown in the upper trace). Vapor cell temperatures are given, with $*$ denoting a shift in the laser beams foci to the entrance of the vapor cell. (upper trace in Fig. 2). The $776 \mathrm{~nm}$ frequency is also monitored with this wavemeter, where the $776 \mathrm{~nm}$ laser detuning $\left(\delta_{776}\right)$ is correspondingly adjusted relative to the $780 \mathrm{~nm}$ laser detuning $\left(\delta_{780}\right)$ to remain on the $5 D_{5 / 2}$ resonance $\left(\delta_{780}=-\delta_{776}\right)$. A linear rise in the blue beam power is observed with increasing $\mathrm{Rb}$ vapor cell temperatures, assuming increasing input powers are available to saturate the FWM process. We examine these saturation levels later in Fig. 3. A threshold $\mathrm{Rb}$ vapor cell temperature of $95^{\circ} \mathrm{C}$ is extrapolated for blue beam generation under our experimental conditions, while $135^{\circ} \mathrm{C}$ is the highest temperature in Fig. 2 , as the blue power begins to decrease above this value. Using $135^{\circ} \mathrm{C}$ as an approximately optimum vapor cell temperature with respect to our maximum input beam powers, we proceeded to empirically test different focusing lens combinations in order to achieve the greatest $420 \mathrm{~nm}$ power. This occurred when the foci of the input laser beams were shifted from the center to the entrance of the vapor cell, denoted by the $*$ data set in Fig. 2 . A $420 \mathrm{~nm}$ beam of $9.1 \mathrm{~mW}$ power was obtained, with input beam diameters of $\simeq 85 \mu \mathrm{m}$ (FWHM) at the entrance of the vapor cell and $\simeq 310 \mu \mathrm{m}$ at the exit, using input laser powers of $P_{780}=390 \mathrm{~mW}$ and $P_{776}=205 \mathrm{~mW}$.

These results can be qualitatively understood by considering the phase-matching conditions, $\vec{k}_{780}+\vec{k}_{776}=$ $\vec{k}_{420}+\vec{k}_{5230}$ for this FWM process, with the wave vector magnitudes $k_{\omega}=\omega / c \cdot n_{\omega}$, where $n_{\omega}$ is the refractive index at the respective frequency. The 780 and $420 \mathrm{~nm}$ optical fields encounter the largest refractive index variation as they are readily absorbed by ground-state $\mathrm{Rb}$ atoms. However, phase matching will be most favorable for minimal changes in $n_{780}$ and $n_{420}$, which Meijer et al. [2] calculated for low laser powers to occur at $780 \mathrm{~nm}$ detunings from 1 to $3 \mathrm{GHz}$. Our data agree well with this result at $\geq 10$ times the input laser intensities. Small changes in the optimum $780 \mathrm{~nm}$ detunings are observed as the $\mathrm{Rb}$ density and input laser intensities are increased. This can be attributed to slight changes in $n_{780}$ and $n_{420}$ which serve to optimize phase matching as the laser detuning moves along the wing of the Doppler broadened ${ }^{85} \mathrm{Rb} 5 S_{1 / 2}(F=2) \rightarrow 5 P_{3 / 2}\left(F^{\prime}\right)$ resonance.

Shifting the input beam focus to the entrance of the vapor cell not only resulted in a significant increase in $420 \mathrm{~nm}$ output power but also in a large shift in the $780 \mathrm{~nm}$ detuning. We note this detuning agrees with results at lower powers, which determined ideal $780 \mathrm{~nm}$ detunings almost directly between the ${ }^{85} \mathrm{Rb}$ ground hyperfine states [ 1,3$]$. Propagation effects such as this have not been well studied; however, similar experiments in sodium highlighted the competition between FWM and amplified spontaneous emission (ASE) in two-photon excitation [15]. It was suggested that when the beam focus is moved toward the entrance of the vapor cell, FWM is initiated more strongly, leading to suppression of upper level populations and associated ASE. Another noticeable propagation effect is an increase in the generated $420 \mathrm{~nm}$ beam power by $5 \%-10 \%$ when blocking the edges of the input laser beams with an aperture. While we would not expect the far wings of the spatially Gaussian beams to contribute to FWM, it is interesting that they 

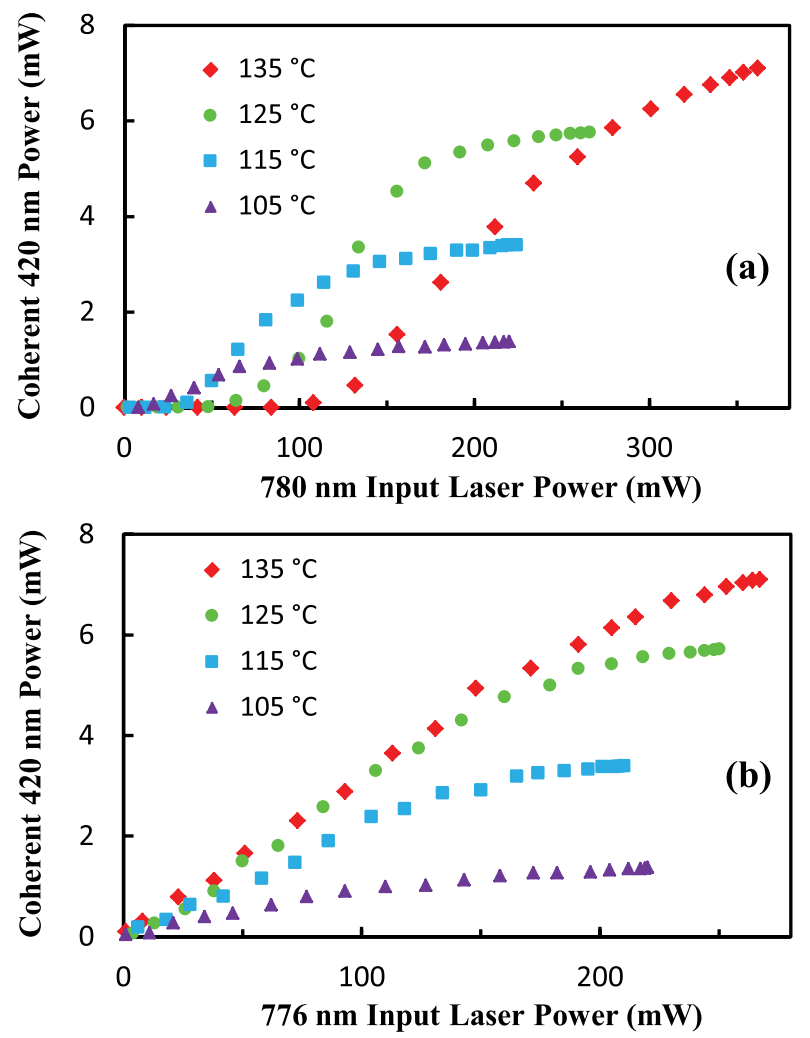

Fig. 3. (a) Output blue power measured while varying $780 \mathrm{~nm}$ input power and holding the $776 \mathrm{~nm}$ input power at its maximum value. (b) Blue power measured while varying the $776 \mathrm{~nm}$ input power and holding the $780 \mathrm{~nm}$ input power at its maximum value.

slightly decrease the process, possibly also due to increased competition from ASE.

Figure 3 illustrates the generated blue beam power as a function of each input beam power for vapor cell temperatures from $105^{\circ} \mathrm{C}$ to $135^{\circ} \mathrm{C}$. As the $780 \mathrm{~nm}$ input power is increased [Fig. 3(a)], we observe both a threshold-like behavior along with saturation of the blue beam power. These characteristics can be described by considering the optical depth (OD) for the $780 \mathrm{~nm}$ beam. The optical depth, $\mathrm{OD}=N \sigma l$, where $N$ is the Rb density, $\sigma$ is the absorption cross section, and $l$ is the length of the vapor cell. In calculating $\sigma$ we take into account the laser intensities and frequency detunings using

$$
\sigma=\frac{\sigma_{0}}{1+4(\Delta / \Gamma)^{2}+\left(I / I_{\mathrm{sat}}\right)}
$$

where $\sigma_{0}$ is the on-resonance cross section, $\Delta$ is the detuning from resonance, $\Gamma$ is the spontaneous decay rate, $I$ is the $780 \mathrm{~nm}$ laser intensity, and $I_{\text {sat }}$ is the saturation intensity for the $5 P_{3 / 2}$ transition. Parameters such as $\sigma_{0}$ and $I_{\text {sat }}$ along with $\mathrm{Rb}$ vapor pressures are taken from the data compiled by Steck [16]. Using the results shown in Fig. 3(a), we extract the $780 \mathrm{~nm}$ laser intensities at the onset of blue light generation and when the blue power saturates. We find, regardless of temperature, the blue power saturates at $\mathrm{OD} \simeq 1$. At higher levels of absorption (corresponding to smaller $780 \mathrm{~nm}$ input powers) the blue power decreases until $\mathrm{OD} \simeq 5-6$ where a blue beam is no longer observed. In this circumstance, the $780 \mathrm{~nm}$ beam is quickly absorbed in the initial length of the vapor cell, at which point the FWM process ceases and the generated $420 \mathrm{~nm}$ beam is also absorbed before it can exit the vapor cell. Figure 3(b) does not show a threshold type of behavior as the $780 \mathrm{~nm}$ beam is already at saturation intensities, allowing the FWM process to propagate throughout the $\mathrm{Rb}$ vapor cell even at low $776 \mathrm{~nm}$ intensities as the $776 \mathrm{~nm}$ beam is weakly absorbed in the Rb vapor. As the above results are consistent across a range of temperatures they may be used to estimate an optimum $\mathrm{Rb}$ density depending upon the laser intensities available.

During analysis of the generated blue beam we also observed a collimated IR beam, measured to be at $1324 \mathrm{~nm}$ using an optical spectrum analyzer. Examining this beam with an InGaAs scanning slit beam profiler showed a Gaussian spatial profile. Previous studies by Zibrov et al. [17] observed a $1.36 \mu \mathrm{m}$ beam which they accounted for by FWM through the $6 S_{1 / 2} \rightarrow 5 P_{3 / 2}$ states. A recent paper confirmed such a forward beam, while also inferring the presence of a $1.32 \mu \mathrm{m}$ beam [18]. In our case the only wavelength detected is $1324 \mathrm{~nm}$, corresponding to the $6 S_{1 / 2} \rightarrow 5 P_{1 / 2}$ transition. Inserting a dichroic beam splitter before the vapor cell which transmits 776-780 nm but reflects $1.3 \mu \mathrm{m}$, revealed this beam is both forward and backward propagating. This suggests a process involving population transfer, such as ASE, and rules out FWM since phase matching dictates a forwardonly beam.

Figure 4 illustrates the dependence of the $1324 \mathrm{~nm}$ beam on the input lasers' frequencies and powers. As phase matching is not required to produce the $1324 \mathrm{~nm}$ beam, Fig. 4(a) shows a much greater range of $780 \mathrm{~nm}$ detunings where this beam is generated compared to the blue beam in Fig. 2. Figure 4(b) demonstrates the power in the forward component of the $1324 \mathrm{~nm}$ beam, which is close to reaching saturation only at the highest input powers. By scanning the $776 \mathrm{~nm}$ laser frequency while simultaneously monitoring both the forward-only $420 \mathrm{~nm}$ beam and the backward-propagating beam at $1324 \mathrm{~nm}$ we obtain the data shown in Fig. 4(c). The $1324 \mathrm{~nm}$ beam is observed over a large detuning range where the blue beam occurs, suggesting cascading population inversions on the $5 D_{5 / 2}, 6 P_{3 / 2}$, and $6 S_{1 / 2}$ states in addition to the FWM process, in agreement with recent results observing the $5.2 \mu \mathrm{m}$ beam emission [18]. By filtering between the blue and IR beams, we measure a forward beam at $1324 \mathrm{~nm}$ of $1.2 \mathrm{~mW}$ when producing $9.0 \mathrm{~mW}$ at $420 \mathrm{~nm}$, which is roughly half the maximum IR power observed. Figure 4(c) also shows the blue and IR beams peaking at different detunings. A complicated interplay between population dynamics and FWM processes is at work, requiring detailed modeling of the coherence and populations among the six atomic states for further understanding of this system.

While we have examined the generated blue and IR beams in terms of the input laser intensities, $\mathrm{Rb}$ density, and laser frequency detunings, a theory is yet to be developed which provides an upper limit on the efficiency of this process. We estimate a $40 \%$ increase in the blue beam power could be achieved simply by using antireflection-coated vapor cells and optimized dichroic 

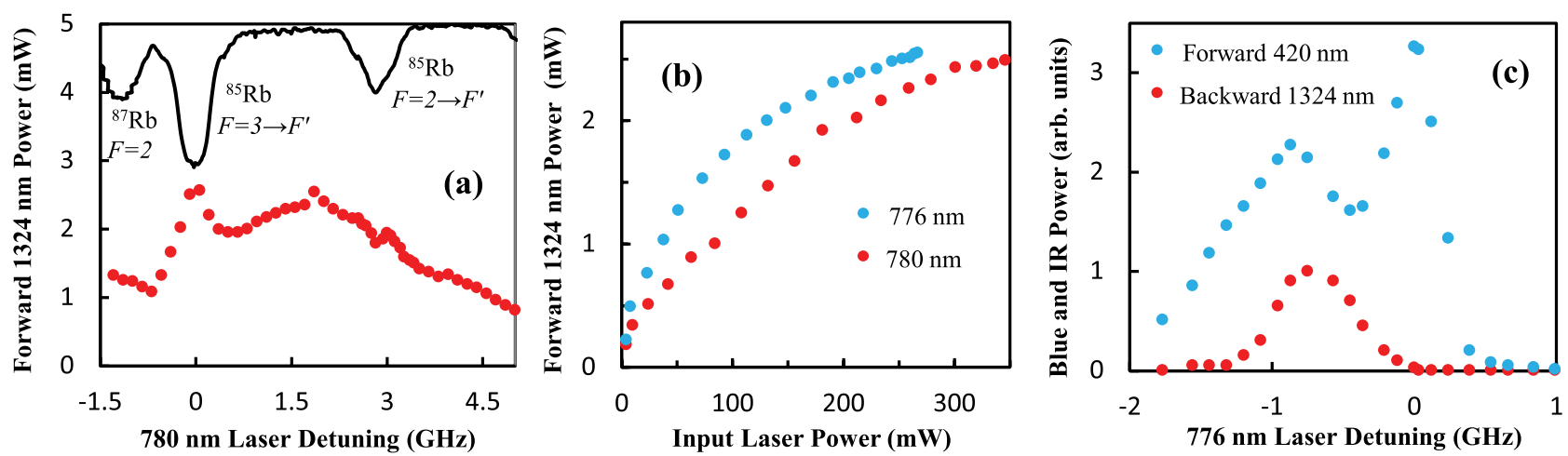

Fig. 4. (a) Forward IR beam power as the input $780 \mathrm{~nm}$ laser is scanned in frequency. The upper curve references our laser detunings to $\mathrm{Rb}$ absorption resonances. (b) Forward IR power dependence on input laser powers (as one input beam power is adjusted the other is held constant at its maximum power). (c) The forward blue and backward IR beams measured simultaneously as the 776 nm frequency is varied.

optics when splitting the output beams. Further advances have the potential to not only increase the efficiency of frequency upconversion and downconversion in $\mathrm{Rb}$ but to generalize the characteristics of this process to other states and atoms that have wavelengths of interest.

The authors gratefully acknowledge support of this research by the Air Force Office of Scientific Research and the National Science Foundation (grant 1206128).

\section{References}

1. A. M. Akulshin, R. J. McLean, A. I. Sidorov, and P. Hannaford, Opt. Express 17, 22861 (2009).

2. T. Meijer, J. D. White, B. Smeets, M. Jeppesen, and R. E. Scholten, Opt. Lett. 31, 1002 (2006).

3. A. Vernier, S. Franke-Arnold, E. Riis, and A. S. Arnold, Opt. Express 18, 17020 (2010).

4. F. E. Becerra, R. T. Willis, S. L. Rolston, and L. A. Orozco, Phys. Rev. A 78, 013834 (2008).

5. A. Akulshin, C. Perrella, G.-W. Truong, R. McLean, and A. Luiten, J. Phys. B 45, 245503 (2012).

6. A. S. Zibrov, M. D. Lukin, L. Hollberg, and M. O. Skully, Phys. Rev. A 65, 051801(R) (2002).

7. A. Akulshin, C. Perrella, G.-W. Truong, A. Luiten, D. Budker, and R. McLean, "Linewidth of collimated wavelengthconverted emission in Rb vapours," arXiv:1301.7117 (2013).
8. A. M. Akulshin, A. A. Orel, and R. J. McLean, J. Phys. B 45, 015401 (2012).

9. E. Brekke and L. Alderson, Opt. Lett. 38, 2147 (2013).

10. C. V. Sulham, G. A. Pitz, and G. P. Perram, Appl. Phys. B 101, 57 (2010).

11. J. T. Schultz, S. Abend, D. Doring, J. E. Debs, P. A. Altin, J. D. White, N. P. Robins, and J. D. Close, Opt. Lett. 34, 2321 (2009).

12. O. S. Heavens, J. Opt. Soc. Am. 51, 1058 (1961).

13. A. M. Akulshin, B. V. Hall, V. Ivannikov, A. A. Orel, and A. I. Sidorov, J. Phys. B 44, 215401 (2011).

14. G. Walker, A. S. Arnold, and S. Franke-Arnold, Phys. Rev. Lett. 108, 243601 (2012).

15. R. W. Boyd, M. S. Malcuit, D. J. Gauthier, and K. Rzazewski, Phys. Rev. A 35, 1648 (1987).

16. D. A. Steck, http://steck.us/alkalidata (Revision 2.1.6, 20 September, 2013).

17. A. S. Zibrov, L. Hollberg, V. L. Velichansky, M. O. Scully, M. D. Lukin, H. G. Robinson, A. B. Matsko, A. V. Taichenachev, and V. I. Yudin, AIP Conf. Proc. 551, 204 (2001).

18. A. Akulshin, D. Budker, and R. McLean, "Directional infrared emission resulting from cascade population inversion and four-wave mixing in Rb vapours," arXiv: 1311.0071 (2013). 